\title{
Determinantes sociodemográficos y clínicos asociados a mortalidad fetal intrauterina en el municipio de Pasto, 2010-2012
}

\author{
Demographic and clinical determinants associated with intrauterine fetal mortality in the \\ municipality of Pasto, 2010 - 2012 \\ Daniel Jurado-Fajardo ${ }^{1,2^{*}}$ orcid.org/0000-0003-0628-0253 \\ Melissa Catherine Chaves-Marcillo3 orcid.org/0000-0001-9876-4065 \\ Annyi Tatiana Belalcázar-Fajardo3 orcid.org/0000-0002-4025-3497 \\ Denny Marcela Achicanoy-Puchana3 ${ }^{3}$ orcid.org/0000-0001-8555-0084 \\ Carlos Andrés Guerrero3 orcid.org/0000-0002-3197-4321 \\ Carmen Eugenia Quiñonez-Arteaga1,4 orcid.org/0000-0003-3495-2272 \\ Luisa Mercedes Bravo-Goyes ${ }^{1,2}$ orcid.org/0000-0002-6440-8289
}

1 Grupo de investigación Salud Publica, Centro de Estudios en Salud - CESUN, Universidad de Nariño. Pasto, Colombia.

2 Programa de Medicina, Facultad de Ciencias de la Salud, Universidad de Nariño, Pasto, Colombia

3 Semillero de investigación Septem, Programa de medicina. Facultad Ciencias de la Salud, Universidad de Nariño. Pasto, Colombia

4 Instituto Departamental de Salud de Nariño. Pasto, Colombia Determinantes sociodemográficos y clínicos asociados a mortalidad fetal intrauterina en el municipio de Pasto 2010-2012. Univ. Salud. 2017;19(2):207-214. DOI: http://dx.doi.org/10.22267/rus.171902.83

\section{Resumen}

Introducción: La mortalidad fetal intrauterina (MFIU) es un problema de salud pública por sus elevadas tasas a nivel mundial y en poblaciones de ingresos medios y bajos. Sin embargo, es un evento poco estudiado y carece de visibilidad en las políticas, planes y programas de salud pública. Objetivo: Analizar los determinantes sociodemográficos y clínicos asociados a la MFIU en Pasto-Colombia. Materiales y métodos: Estudio analítico observacional con 88 muertes fetales como casos y 88 nacidos vivos como controles, ocurridas en hospitales de tercer nivel en el municipio de Pasto-Colombia durante 2010-2012, para determinar la relación entre mortalidad fetal, condiciones clínicas (complicaciones del embarazo, edad gestacional, peso al nacer, controles prenatales, antecedentes obstétricos, tóxicos o aborto) y sociodemográficas (edad, etnia, ocupación, estado civil, estrato, zona de residencia, escolaridad, paridad, condición de desplazamiento, embarazo planeado). Resultados: Se identificó que el riesgo de mortalidad fetal es significativamente menor con el incremento de la edad gestacional (OR ajustado=0,76 IC95\% 0,62; 0,93) y el peso al nacer (OR ajustado=0,99 IC95\% 0,98; 0,99). Otras variables clínicas y sociodemográficas no se asociaron. Conclusión: Los resultados proveen evidencia para la planificación de planes de intervención que prioricen a mujeres cuyo feto tenga un peso inferior al normal y un riesgo de nacimiento prematuro.

Palabras clave: Determinantes, mortalidad fetal intrauterina, factores clínicos, factores sociodemográficos. (Fuente: DeCS, Bireme).

\footnotetext{
*Autor de correspondencia

Daniel Jurado Fajardo

e-mail: danieljuradof@gmail.com
} 


\begin{abstract}
Introduction: Intrauterine fetal mortality (IUFM) is a public health problem because of its high rates worldwide and in low-and middle-income populations. However, it is a little-studied event and lacks visibility in public health policies, plans and programs. Objective: To analyze the sociodemographic and clinical determinants associated with IUFM in Pasto-Colombia. Materials and methods: A study, that includes 88 fetal deaths as cases and 88 live births as controls occurred in third level hospitals in Pasto-Colombia during 2010 and 2012, was carried out to determine the relationship between fetal mortality, clinical conditions (complications of pregnancy, gestational age, birth weight, prenatal controls, pathological and toxic medical history, or abortion) and sociodemographic conditions (age, ethnicity, occupation, marital status, stratum, area of residence, schooling, parity, displacement condition, planned pregnancy). Results: It was identified that the risk of fetal mortality is significantly lower with the increase in gestational age (OR ajustado $=0.76$ IC95\% 0.62; 0.93) and birth weight (OR ajustado $=0.99$ IC95\% 0.98; 0.99). Other clinical and sociodemographic variables were not associated. Conclusion: The results provide evidence for planning intervention plans that prioritize women whose fetus has a lower-than-normal weight and a risk of premature birth.
\end{abstract}

Keywords: Determinants, intrauterine fetal death, clinical factors, sociodemographic factors. (Source: DeCS, Bireme).

\section{Introducción}

La Mortalidad Fetal Intrauterina tardía hace referencia a las muertes producidas durante el embarazo después de la semana veintiocho de gestación, con un peso al nacer $\geq 1000$ gr y de talla $\geq 35 \mathrm{~cm}^{(1)}$, se ha convertido en un problema de salud pública por su alta tasa de mortalidad registrada a nivel mundial, con más de 2,64 millones de muertes para el 2012, de las cuales el $98 \%$ ocurre en países de bajos ingresos(2-5). En conjunto con la mortalidad perinatal, constituyen un medidor de la calidad de la atención que se brinda a la madre y su hijo(6).

La mortalidad fetal se origina a partir de las desigualdades e inequidades de la población, tanto en el ámbito local como internacional. Mundialmente existen diferencias significativas entre las tasas de mortalidad fetal según la capacidad de desarrollo de los países, evidenciándose que aquellos con pobreza extrema tienen mayores tasas de mortalidad fetal (29 por 1000 nacidos vivos) con respecto a aquellos de ingresos altos $(3,1$ por 1000 nacidos vivos); igualmente se identifica que son prácticamente tres veces más altas entre etnias diferentes que en el resto de la población, considerando que las mujeres indígenas tienen un riesgo casi dos veces más alto que las no indígenas $(4,7)$.
Otras condiciones y factores de riesgo que se desarrollan paralelas al periodo de gestación, se han detectado como determinantes, tales como: lugar geográfico de residencia, nivel educativo bajo, estrato socioeconómico, paridad, estado; comportamentales asociados a la obesidad, tabaquismo o alcoholismo antes y durante el embarazo; culturales como las costumbres y tradiciones de la atención de partos en casa, parto de rodillas, ritos y ceremonias; y otros asociados al acceso al derecho a la salud como la inclusión en controles prenatales y programas de maternidad. Sin embargo, la información que se tiene actualmente acerca de las causas que generan la mortalidad fetal no es concluyente ${ }^{(8,9)}$.

En Colombia, no existe información suficiente que permita identificar la dimensión de este fenómeno y las características de sus determinantes para ser documentados e intervenidos, reduciendo así la importancia de este evento en el país(10). Adicionalmente, las estrategias para su intervención no se encuentran específicamente registradas en políticas mundiales como los objetivos de desarrollo del milenio, el seguimiento por parte de la ONU o las mediciones de la carga mundial de morbilidad, tampoco se tienen en cuenta en programas nacionales como el plan decenal de salud pública, y dispone de escasos fondos para su investigación. Siendo así, un fenómeno que carece de visibilidad en las políticas, planes y programas de salud pública, las cuales se han 
centrado únicamente en la supervivencia después del nacimiento y no durante la gestación en donde se presenta la mayoría de las muertes $(1,5,11)$. Por lo tanto, es importante continuar el proceso de investigación en esta área, debido a la escasez de conocimiento encontrada a nivel regional y nacional, donde se requiere una caracterización adecuada de los casos de mortalidad fetal y un estudio de sus determinantes, para tomar medidas de prevención necesarias en los planes y programas de salud, priorizando poblaciones vulnerables desde el punto de vista clínico o sociodemográfico(5,9). Este estudio tiene como objetivo analizar los determinantes asociados a la MFUI en el municipio de Pasto-Colombia.

\section{Materiales y métodos}

Se realizó un estudio observacional analítico de casos y controles sobre mortalidad fetal intrauterina y sus determinantes. El estudio se desarrolló con los nacimientos ocurridos en el periodo de enero de 2010 a diciembre de 2012 en hospitales de tercer nivel de complejidad que brindan atención médica a población abierta, de todos los regímenes de salud y han reportado la mayor proporción MFIU en el municipio de Pasto para ese periodo $(75 \%)$.

Se consideraron como casos $(\mathrm{N}=88)$ a todas los nacidos muertos mayores de 28 semanas de gestación y como controles $(\mathrm{n}=88)$ los nacidos vivos. Se excluyeron las muertes no certificadas por personal médico y sin historia clínica (25\%). La selección de los controles fue aleatoria a partir de los todos nacimientos ocurridos $(\mathrm{N}=12.373)$ en las instituciones durante el mismo periodo; se seleccionó un control por cada caso.

Se analizaron condiciones sociodemográficas de la madre (edad materna, nivel de educación, etnia, ocupación, régimen, zona de residencia, estado civil, condición de desplazamiento) y clínicas del binomio madre-hijo (antecedentes obstétricos, tóxicos y aborto previo, complicaciones en el embarazo, parto asistido, control prenatal, paridad, planeación del embarazo, fallo de método anticonceptivo, edad gestacional y peso al nacer). La información fue recolectada de la historia clínica e historia clínica y perinatal - CLAP (Centro Latinoamericano de Perinatología) de cada una de las gestantes. Los datos fueron resumidos según la naturaleza de las variables: cualitativas con medidas de frecuencia; cuantitativa con medidas de tendencia central y de dispersión.

Se aplicaron modelos de regresión logística para analizar la asociación y fuerza de asociación (Odds Ratio OR) entre la MFIU y las condiciones sociodemográficas y clínicas de la gestante. Todas las pruebas se realizaron con un nivel de confianza del 95\% en el programa SPSS versión 22.

\section{Consideraciones éticas}

Según la Resolución 8430 de 1993 del Ministerio de Salud por la cual se establecen las normas científicas, técnicas y administrativas para la investigación en salud este estudio se clasifica en la categoría "sin riesgo", teniendo en cuenta que es un estudio sin intervención y que los datos se obtuvieron de la revisión de bases y datos e historias clínicas cumpliendo con las normas de confidencialidad. El proyecto contó con el aval de las instituciones de salud participantes: Hospital Universitario Departamental de Nariño, Fundación Hospital San Pedro y del Comité Curricular de la Facultad de Ciencias de la Salud de la Universidad de Nariño.

\section{Resultados}

En la población total de individuos analizados en este estudio ( $\mathrm{N}=176)$, el mayor porcentaje de gestantes tenía un nivel de escolaridad básica secundaria (52.3\%), habitaban en la zona urbana $(59,1 \%)$, eran de raza mestiza $(77,3 \%)$, pertenecían al régimen subsidiado $(88,6 \%)$, estado civil con pareja $(64,2 \%)$, no planearon el embarazo (66,5\%), no utilizaron método anticonceptivo $(88,6 \%)$, sin condición de desplazamiento $(96,0 \%)$, no presentaron antecedentes tóxicos (96,0\%) ni antecedentes obstétricos (88,6\%), tenían menos de 6 controles prenatales $(65,9 \%)$ y un paridada mayor o igual a 3 (91,5\%), ni complicaciones durante el embarazo $(67,0 \%)$. El promedio de edad materna 
al momento del estudio fue de 25 años (DE: $7,34)$, el promedio de edad gestacional fue de 36,5 semanas (DE: 3,24) y el promedio de peso al nacer fue de 2592 gr (DE: 864).

De los 88 casos de MFIU, se identificó que las características más frecuentes del binomio madre-hijo fueron: escolaridad básica secundaria $(51,1 \%)$, raza mestiza $(78,4 \%)$, residencia urbana $(52,3 \%)$, régimen subsidiado $(93,2 \%)$, convivencia con pareja $(65,9 \%)$, embarazo no planeado $(69,3 \%)$, carencia de uso de métodos anticonceptivos $(85,2 \%)$, sin condición de desplazamiento $(94,3 \%)$, ausencia de antecedentes tóxicos $(95,5 \%)$ y obstétricos $(85,2 \%)$, número de controles prenatales menor a $6(75 \%)$ y una paridad menor a $3(88,6 \%)$, sin antecedentes de aborto previo (79,5\%), ni complicaciones durante el embarazo $(60,2 \%)$, y edad materna $\mu=24$ años (DE: 6,64), edad gestacional $\mu=34,9$ semanas (DE: 3,46$)$ y peso al nacer $\mu=2.025$ gr (389) (Tabla 1 ).

Tabla1. Características sociodemográficas y clínicas de las muertes fetales y nacidos vivos en el municipio de Pasto 2010 -2012

\begin{tabular}{|c|c|c|c|c|c|c|}
\hline \multirow{2}{*}{ Grupo } & \multirow{2}{*}{ Variable } & \multirow{2}{*}{ Categoría } & \multicolumn{2}{|c|}{ Muerte fetal (Casos) } & \multicolumn{2}{|c|}{ Nacidos Vivos (Controles) } \\
\hline & & & $\mathrm{N}=\mathbf{8 8}$ & $\%$ & $n=88$ & $\%$ \\
\hline \multirow{14}{*}{ 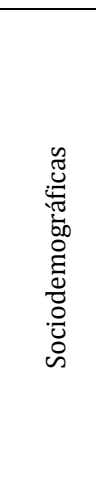 } & \multirow{3}{*}{ Escolaridad } & Universitaria & 1 & 1,1 & 4 & 4,5 \\
\hline & & Básica primaria & 42 & 47,7 & 36 & 40,9 \\
\hline & & Básica secundaria & 45 & 51,1 & 47 & 53,4 \\
\hline & \multirow{3}{*}{ Zona de residencia } & Urbano & 46 & 52,3 & 58 & 65,9 \\
\hline & & Rural & 42 & 47,7 & 30 & 34,1 \\
\hline & & Blanco & 3 & 3,4 & 9 & 10,2 \\
\hline & \multirow[t]{2}{*}{ Etnia } & Mestizo & 69 & 78,4 & 67 & 76,1 \\
\hline & & Otro & 16 & 18,2 & 12 & 13,6 \\
\hline & \multirow{2}{*}{$\begin{array}{l}\text { Régimen de afiliación a } \\
\text { salud }\end{array}$} & Contributivo & 6 & 6,8 & 14 & 15,9 \\
\hline & & Subsidiado & 82 & 93,2 & 74 & 84,1 \\
\hline & \multirow{2}{*}{ Estado civil } & Con pareja & 58 & 65,9 & 55 & 62,5 \\
\hline & & Sin pareja & 30 & 34,1 & 33 & 37,5 \\
\hline & \multirow{2}{*}{$\begin{array}{l}\text { Condición de } \\
\text { desplazamiento }\end{array}$} & No & 83 & 94,3 & 86 & 97,7 \\
\hline & & $\mathrm{Si}$ & 5 & 5,7 & 2 & 2,3 \\
\hline \multirow{20}{*}{ 苞 } & \multirow{2}{*}{ Antecedente tóxico } & No & 84 & 95,5 & 85 & 96,6 \\
\hline & & $\mathrm{Si}$ & 4 & 4,5 & 3 & 3,4 \\
\hline & \multirow{2}{*}{ Antecedente obstétrico } & No & 75 & 85,2 & 81 & 92 \\
\hline & & $\mathrm{Si}$ & 13 & 14,8 & 7 & 8 \\
\hline & \multirow{2}{*}{ Paridad } & Menor o igual 3 & 78 & 88,6 & 83 & 94,3 \\
\hline & & Mayor a 3 & 10 & 11,4 & 5 & 5,7 \\
\hline & \multirow{2}{*}{$\mathrm{N}^{\circ}$ Control prenatal } & Mayor a 6 & 22 & 25 & 38 & 43,2 \\
\hline & & Menor o igual a 6 & 66 & 75 & 50 & 56,8 \\
\hline & \multirow{3}{*}{ Anticonceptivo } & Hormonal & 11 & 12,5 & 6 & 6,8 \\
\hline & & No usaba & 75 & 85,2 & 81 & 92 \\
\hline & & Barrera & 2 & 2,3 & 1 & 1,1 \\
\hline & \multirow{2}{*}{ Embarazo planeado } & $\mathrm{Si}$ & 27 & 30,7 & 32 & 36,4 \\
\hline & & No & 61 & 69,3 & 56 & 63,6 \\
\hline & \multirow{2}{*}{$\begin{array}{l}\text { Complicaciones en el } \\
\text { embarazo }\end{array}$} & $\mathrm{Si}$ & 35 & 39,8 & 23 & 26,1 \\
\hline & & No & 53 & 60,2 & 65 & 73,9 \\
\hline & \multirow{2}{*}{ Aborto } & $\mathrm{Si}$ & 18 & 20,5 & 16 & 18,2 \\
\hline & & No & 70 & 79,5 & 86 & 97,7 \\
\hline & \multicolumn{2}{|c|}{ Edad materna en años $\mu / \bar{X}$ y DE) } & 24,49 & 6,64 & 27,2 & 7,851 \\
\hline & \multicolumn{2}{|c|}{ Edad Gestacional en semanas $(\mu / \bar{X}$ y DE $)$} & 34,94 & 3,46 & 38,0 & 2 \\
\hline & \multicolumn{2}{|c|}{ Peso de Nacimiento en gr $(\mu / \bar{x}$ y DE $)$} & 2025 & 389 & 2992,2 & 551,9 \\
\hline
\end{tabular}

$\mathrm{N} / \mathrm{n}$ : número de individuos; $\mu / \overline{\mathrm{X}}$ : promedio poblacional/promedio muestral; DE: desviación estándar

En la población de controles o de nacidos vivos, se identificó que las características del binomio madre-hijo más frecuentes fueron: escolaridad básica secundaria $(53,4 \%)$, residencia urbana $(65,9 \%)$, raza mestiza $(76,1 \%)$, régimen subsidiado $(84,1 \%)$, sin condición de desplazamiento $(97,7 \%)$, convivencia con pareja $(62,5 \%)$, embarazo no planeado $(63,6 \%)$, no usaban método anticonceptivo (92\%), sin antecedente tóxicos $(96,6 \%)$, ni obstétricos (92\%), un número de controles prenatales menor o igual a $6(56,8 \%)$ y una paridad menor 
o igual a $3(94,3 \%)$, no presentaron abortos previos $(97,7 \%)$ ni complicaciones durante el embarazo (73,9\%), edad materna $\bar{X}=27$ años (DE: 7,85), edad gestacional $\bar{x}=38$ semanas (DE: 2) y peso al nacer $\bar{x}=2992$ gr (DE: 551,9 ).

Algunas de las características como la ocupación, estrato y parto asistido fueron constantes, es decir, no presentaron variabilidad tanto en los casos como en los controles, por lo tanto no se continuó con su análisis.
En la regresión logística bivariada se encontró asociación $(\mathrm{p}<0,25$ según el criterio de HosmerLemeshow) entre la MFIU y la edad materna, edad gestacional, peso al nacimiento, antecedentes médicos, paridad, número de controles prenatales, complicaciones durante el embarazo, escolaridad, zona de residencia, etnia y régimen de afiliación (Tabla 2 ). En el análisis multivariado se conservó únicamente la asociación $(\mathrm{p}<0,05)$ entre MFIU y peso al nacimiento y edad gestacional.

Tabla 2. Efecto de las condiciones clínicas y sociodemográficos en la mortalidad fetal intrauterina

\begin{tabular}{|c|c|c|c|c|c|c|}
\hline \multicolumn{3}{|c|}{ Variables de estudio } & $\begin{array}{l}\text { P-Valor } \\
\text { crudo }\end{array}$ & $\begin{array}{l}\text { OR Crudo } \\
\text { (IC 95\%) }\end{array}$ & $\begin{array}{c}\text { P-Valor } \\
\text { Ajustado }\end{array}$ & $\begin{array}{l}\text { OR Ajustas } \\
\text { (IC 95\%) }\end{array}$ \\
\hline \multirow{14}{*}{ 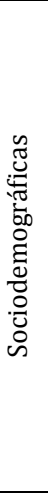 } & \multirow{3}{*}{ Escolaridad } & Universitaria & 1 & 1 & 1 & 1 \\
\hline & & Básica primaria & $0,16^{*}$ & $4,87(0,54 ; 43,64)$ & 0,76 & $1,53(0,1 ; 23,58)$ \\
\hline & & Básica secundaria & $0,22^{*}$ & $3,94(0,44 ; 35,07)$ & 0,96 & $0,93(0,06 ; 14,75)$ \\
\hline & \multirow{2}{*}{ Zona de residencia } & Urbano & 1 & 1 & 1 & 1 \\
\hline & & Rural & $0,06^{*}$ & $1,75(0,97 ; 3,14)$ & 0,47 & $1,33(0,61 ; 2,89)$ \\
\hline & \multirow{3}{*}{ Etnia } & Blanco & 1,00 & 1 & 1 & 1 \\
\hline & & Mestizo & $0,11^{*}$ & $2,95(0,78 ; 11,15)$ & 0,66 & $1,46(0,28 ; 7,53)$ \\
\hline & & Otro & $0,08^{*}$ & $3,81(0,87 ; 16,67)$ & 0,63 & $1,59(0,25 ; 10,21)$ \\
\hline & \multirow{2}{*}{$\begin{array}{l}\text { Régimen de afiliación } \\
\text { a salud }\end{array}$} & Contributivo & 1,00 & 1 & 1 & 1 \\
\hline & & Subsidiado & $0,06^{*}$ & $2,57(0,96 ; 6,9)$ & 0,22 & $2,68(0,55 ; 12,98)$ \\
\hline & \multirow{2}{*}{ Estado civil } & Con pareja & 1,00 & 1 & - & - \\
\hline & & Sin pareja & 0,58 & $0,84(0,46 ; 1,53)$ & - & - \\
\hline & \multirow{2}{*}{$\begin{array}{l}\text { Condición de } \\
\text { desplazamiento }\end{array}$} & No & 1,00 & 1 & - & - \\
\hline & & $\mathrm{Si}$ & 0,39 & $1,38(0,66 ; 2,91)$ & - & - \\
\hline \multirow{20}{*}{ 窎 } & \multirow{2}{*}{ Antecedente tóxico } & No & 1 & 1 & - & - \\
\hline & & $\mathrm{Si}$ & 0,82 & $1,18(0,29 ; 4,87)$ & - & - \\
\hline & \multirow{2}{*}{$\begin{array}{l}\text { Antecedente } \\
\text { obstétrico }\end{array}$} & No & 1 & 1 & 1 & 1 \\
\hline & & $\mathrm{Si}$ & $0,13^{*}$ & $2,04(0,8 ; 5,17)$ & 0,14 & $2,54(0,73 ; 8,84)$ \\
\hline & \multirow{2}{*}{ Paridad } & Menor o igual 3 & 1 & 1 & 1 & 1 \\
\hline & & Mayor a 3 & $0,12^{*}$ & $2,44(0,8 ; 7,42)$ & 0,41 & $0,55(0,13 ; 2,27)$ \\
\hline & \multirow{2}{*}{$\mathrm{N}^{\circ}$ Control prenatal } & Mayor a 6 & 1 & 1 & 1 & 1 \\
\hline & & Menor o igual a 6 & $0,01^{*}$ & $0,43(0,22 ; 0,81)$ & 0,48 & $1,35(0,59 ; 3,04)$ \\
\hline & \multirow{3}{*}{ Anticonceptivo } & Hormonal & 1 & 1 & - & - \\
\hline & & No usaba & 0,31 & $0,5(0,13 ; 1,93)$ & - & - \\
\hline & & Barrera & 0,74 & $1,6(0,1 ; 24,7)$ & - & - \\
\hline & \multirow{2}{*}{ Embarazo planeado } & $\mathrm{Si}$ & 1 & 1 & - & - \\
\hline & & No & 0,99 & $1(0,53 ; 1,91)$ & - & - \\
\hline & \multirow{2}{*}{$\begin{array}{l}\text { Complicaciones en el } \\
\text { embarazo }\end{array}$} & $\mathrm{Si}$ & 1 & 1 & 1 & 1 \\
\hline & & No & $0,05^{*}$ & $1,81(0,98 ; 3,34)$ & 0,65 & $1,22(0,52 ; 2,89)$ \\
\hline & \multirow{2}{*}{ Aborto } & $\mathrm{Si}$ & 1 & 1 & - & - \\
\hline & & No & 0,39 & $1,38(0,66 ; 2,91)$ & - & - \\
\hline & \multicolumn{2}{|c|}{ Edad materna en años (X y DE) } & $0,01^{*}$ & $1,05(1,01 ; 1,1)$ & 0,84 & $1,01(0,95 ; 1,07)$ \\
\hline & \multicolumn{2}{|c|}{ Edad Gestacional en semanas (X y DE) } & $0,00^{*}$ & $0,67(0,59 ; 0,76)$ & $0,01^{* *}$ & $0,76(0,62 ; 0,93)$ \\
\hline & \multicolumn{2}{|c|}{ Peso de Nacimiento en gramos (X y DE) } & $0,00^{*}$ & $0,99(0,98 ; 0,99)$ & $0,01^{* *}$ & $0,99(0,98 ; 0,99)$ \\
\hline
\end{tabular}

* Variable estadísticamente significativa (p-valor Wald<0,25) según el criterio de Hosmer-Lemeshow

** Variable estadísticamente significativa ( $p$-valor Wald $<0,05$ )

El riesgo de mortalidad disminuye $1 \%$ por cada gramo de peso que aumente el feto (OR ajustado=0,99 IC95\% 0,98; 0,99 ) y $24 \%$ por cada semana de gestación que aumente (OR ajustado=0,76 IC95\% 0,62; 0,93). Por lo tanto, el peso al nacer como la edad gestacional actúan como factores protectores al disminuir el riesgo de MFIU.

A pesar que las variables escolaridad y etnia no son estadísticamente significativas $(\mathrm{p}<0,05)$, al analizar los intervalos de confianza se evidencia 
una tendencia de incremento de riesgo de MFIU (un bajo nivel de escolaridad y etnia diferente a la mestiza o blanca presentarían 1,5 veces más riesgo de MFIU) que podría asumirse como de significancia clínica y que probablemente al ampliar la población de estudio se podría concluir sobre su asociación(12).

\section{Discusión}

Durante la gestación, tanto madre como feto se encuentran expuestos a factores de riesgo que de manera directa o indirecta influyen en el desarrollo normal del embarazo, estos amenazan la salud, la vida y en gran parte conllevan a desenlaces fatales; sin embargo, la información que se tiene actualmente no es del todo concluyente acerca de las causas que generan la mortalidad fetal, razón por la cual aún no se ha considerado como un problema prioritario de salud pública, carece de estrategias de intervención y sus repercusiones son serias en la vida familiar(8,13). Generalmente, se considera que es un evento determinado por un componente clínico y patológico de la madre o del feto. Recientemente se ha intentado buscar una respuesta en determinantes demográficos y socioeconómicos que podrían llegar a restringir el desarrollo fetal intrauterino normal ocasionando la muerte, y a pesar de la existencia de varios estudios relacionados con la MFIU, son pocos los que han encontrado una asociación con estos factores. $(9,14)$.

En el presente estudio se consideraron todos los posibles factores sociodemográficos, además de los clínicos, que la literatura reporta como posibles determinantes. Sin embargo se realizó en hospitales de tercer nivel de complejidad en donde la mayoría de la atención a las gestantes se centra a aquellas que tienen un alto riesgo obstétrico, principalmente definido por características clínicas; de ello se puede deducir que posiblemente el factor clínico se superpone a otros factores sociales y no permite un análisis real del contexto demográfico y socioeconómico en el cual se ha desarrollado la gestación. Por otra parte, no se pudo analizar condiciones socioeconómicas como la ocupación y el estrato, constituyéndose en otra limitación del estudio.
Se identificaron otras investigaciones que obtuvieron resultados similares a los encontrados en este estudio; un meta análisis realizado por Berhan et al., que incluye 14 estudios realizados en 11 países entre ellos Brasil, Tailandia, Nigeria y Bangladesh, concluyó que la mortalidad perinatal presenta un riesgo estadísticamente significativo con respecto a la edad materna, donde madres adolescentes y una baja edad gestacional tendrían mayor riesgo; adicionalmente no se encontró una verdadera influencia para determinantes socioeconómicos estudiados, aunque su posible relación no puede ser descartada en combinación con otros factores, debido a la metodología de muchos de los estudios de pequeña escala y se recomienda profundizar los estudios en poblaciones de mayor tamaño(15).

Estudios latinoamericanos realizados por Linares et al., demuestran que el nivel de educación fue similar tanto en casos como controles, en donde la baja escolaridad no fue un factor de riesgo para MFIU, pero se encontró gran correlación entre causas clínicas prevenibles (obesidad, hipertensión en el embarazo, consumo de tóxicos) que pudieron haber sido identificadas e intervenidas oportunamente durante los controles prenatales(16). Como contraste en el estudio de Pandero et al. determinó la existencia de una asociación clínica y estadística entre madres de bajo nivel educativo y las muertes fetales(17).

Pandero et al. y el presente estudio coinciden en que existe una asociación entre edad gestacional y las muertes ante parto, en donde los casos de muertes presentaron menos de 37 semanas de gestación, es decir ocurrieron en embarazos pretérmino a diferencia de los grupos controles $(16,17)$. Este aspecto asociado a un bajo peso al nacimiento (entre 2000 a $2500 \mathrm{gr}$ ) y complicaciones durante el embarazo, demostraron un incremento de cinco veces mayor riesgo de morir antes de nacer $y$ determinantes socioeconómicos detectados durante las visitas prenatales como madres primigestantes que contaban con pareja, influían en la detección e intervención de otros factores asociados a un gestación de alto riesgo (17-20). 
Universidad y Salud

Bukowski et al., realizaron estudios en 59 centros hospitalarios de Estados Unidos y demostraron que la restricción de crecimiento fetal tenía una significancia estadística alta al relacionarse con mortalidad perinatal $(p=0,001)$ y por la cual se incrementaba el riesgo tres veces más que en fetos con adecuado peso(21).

Surekha et al. desarrollaron a cabo estudios en India durante el mismo periodo de tiempo, encontrando que la mortalidad perinatal fue más frecuente en la zona rural, en una situación socioeconómica baja y ninguna de las mujeres pertenecían a la clase social media alta o superior; la mayoría de ellas no tenían educación y solo un $1 \%$ alcanzaron a graduarse, resultados que en estos aspectos difieren del presente estudio, pero que si manifiestan una relación concluyendo que la disminución de peso fetal es inversamente proporcional a la muerte perinatal, es decir, a mayor peso adquirido por el feto durante la gestación, menor será su riesgo de morir intrautero(8).

\section{Conclusiones}

Estos resultados son importantes porque reafirman la asociación entre MFIU las variables clínicas como el peso del feto y la edad gestacional. Las mujeres cuyo feto tenga un peso inferior al normal según la edad gestacional y tenga riesgo de nacimiento prematuro deben ser atendidas prioritariamente para contribuir a la disminución de muerte fetal intrauterino. No existe relación contundente entre factores sociodemográficos con MFIU, se considera profundizar y ampliar el estudio incluyendo otras instituciones de salud en el municipio.

\section{Agradecimientos}

Los autores agradecen al Programa de Medicina de la Facultad Ciencias de la Salud y al Centro de estudios en Salud Universidad de Nariño (CESUN) por su apoyo en la ejecución de esta investigación y al Hospital Universitario Departamental de Nariño y a la Fundación Hospital San Pedro por permitir el acceso a la información.

\section{Conflicto de intereses}

Los autores declarar no tener conflicto de intereses.

\section{Referencias}

1. Frøen JF, Cacciatore J, McClure EM, Kuti O, Hakeem JA, Islam M, et al. Stillbirths: Why they matter. Lancet. 2011; 377(9774): p1353-1366.

2. United Nations, Department of Economic and Social Affairs, Population Division. Word Population Prospects: The 2012 Revision. New York (EEUU): UN, D; 2013.

3. McClure EM, Pasha O, Goudar S, Chomba E, Garces A, Tshefu A, et al. Epidemiology of stillbirth in low-middle income countries: A Global Network Study. Acta Obstet Gynecol Scand. 2011; 90(12):1379-85.

4. Goldenberg R, Saleem S, Pasha O, Harrison M, Mcclure E. Reducing stillbirths in low-income countries. Acta obstetricia et gynecologica Scandinavica. 2015; 95(2):135-43.

5. Cousens S, Blencowe H, Stanton C, Chou D, Ahmed S, Steinhardt L, et al. National, regional, and worldwide estimates of stillbirth rates in 2009 with trends since 1995: a systematic analysis. Lancet. 2011; 377(9774):1319-1330

6. Stephansson O, Dickman P, Johansson A, Cnattingius S. The influence of socioeconomic status on stillbirth risk in Sweden. Int J Epidemiol. 2001; 30(6):1296-13.

7. Ravelli AC, Tromp M, Eskes M, Droog JC, van der Post JA, Jager KJ, et al. Ethnic differences in stillbirth and early neonatal mortality in The Netherlands. J Epidemiol Community Health. 2011; 65(8): 696-701.

8. Surekha T, Kumar N. Socio demographic factors affecting perinatal mortality-A study in a rural setup. IOSR Journal of Pharmacy. 2012; 2(4):43-46.

9. Molina S, Alfonso D. Muerte fetal anteparto: ¿Es una condición prevenible?. Univ. Méd. Bogotá. 2010; 51(1): 59-73.

10. Guardela L, Barrios I. Colombia: ¿en la vía del desarrollo sostenible?. Revista de derecho. 2006; 26:110-136.

11. Organización de Naciones Unidas. Objetivos de Desarrollo del Milenio [Internet]. Ginebra (Suiza): ONU [citado Agosto 2017]. Disponible en: http://www.un.org/es/millenniumgoals/

12. Escrig J, Millares J, Martinez D, Rivadulla I. Intervalos de confianza: por qué usarlos. Cir Esp. 2007; 81(3):121-5.

13. Ticona M, Huanco D. Factores de riesgo de la mortalidad perinatal en hospitales del Ministerio de Salud del Perú. Rev Cubana Obstet Ginecol. 2011; 37(3):432-443.

14. Lawn JE, Blencowe H, Pattinson R, Cousens S, Kumar R, Ibiebele I, et al. Stillbirths: Where? When? Why? How to make the data count?. Lancet. 2011; 377(9775):144863.

15. Berhan, Y, Berhan A. A Meta-Analysis of SocioDemographic Factors for Perinatal Mortality in Developing Countries: A Subgroup Analysis of the 
National Surveys and Small Scale Studies. Ethiop J Health Sci. 2014; 24(0Suppl):41-54.

16. Linares J, Poulsen R. Muerte Fetal In Útero: Etiología y factores asociados en un Hospital Regional de Antofagasta, Chile. CIMEL. 2006; 11(2): 7-10.

17. Panduro JG, Pérez J, Panduro MG, Castro J, Vázquez M. Factores de riesgo prenatales en la muerte fetal tardía; Hospital Civil de Guadalajara, México. Rev. chil. obstet. ginecol. 2011; 76(3): 169-174.

18. Aquino T, Bezerra MJ, Wanick S, Cardoso L. Fatores de risco para a mortalidade perinatal no Recife, Pernambuco, Brasil, 2003. Cad. Saúde Pública. 2007; 23(12): 2853-2861.

19. Moreno N. Factores que se asocian a la muerte fetal intrauterina presentados en el hospital "María Auxiliadora" del Cono Sur De Lima-2011. Rev. Peri. Enferm. 2012; 8(2): 56-66.

20. De Pardo E, Arandia R. Factores perinatales asociados a morbimortalidad neonatal. Gac Med Bol. 2008; 31(1): 5-13.

21. Bukowski, R., Hansen, N. I., Willinger, M., Reddy, U. M., Parker, C. B., Pinar, H., \& Koch, M. A. Fetal growth and risk of stillbirth: a population-based case-control study. PLoS Med. 2014; 11(4): e1001633. 